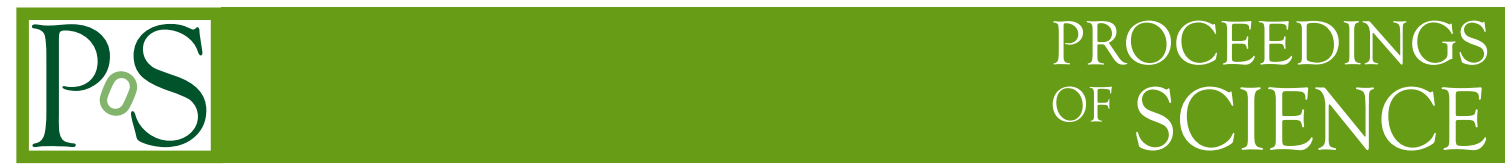

\title{
Top Quark Physics
}

\author{
Markus Schulze* \\ Humboldt-Universität zu Berlin \\ E-mail: markus.schulze@physik.hu-berlin.de
}

This plenary talk briefly reviews recent theory progress for top quark production at the LHC and the impact of precision calculations on physics analyses in the Standard Model and beyond.

Sixth Annual Conference on Large Hadron Collider Physics (LHCP2018)

4-9 June 2018

Bologna, Italy

${ }^{*}$ Speaker. 


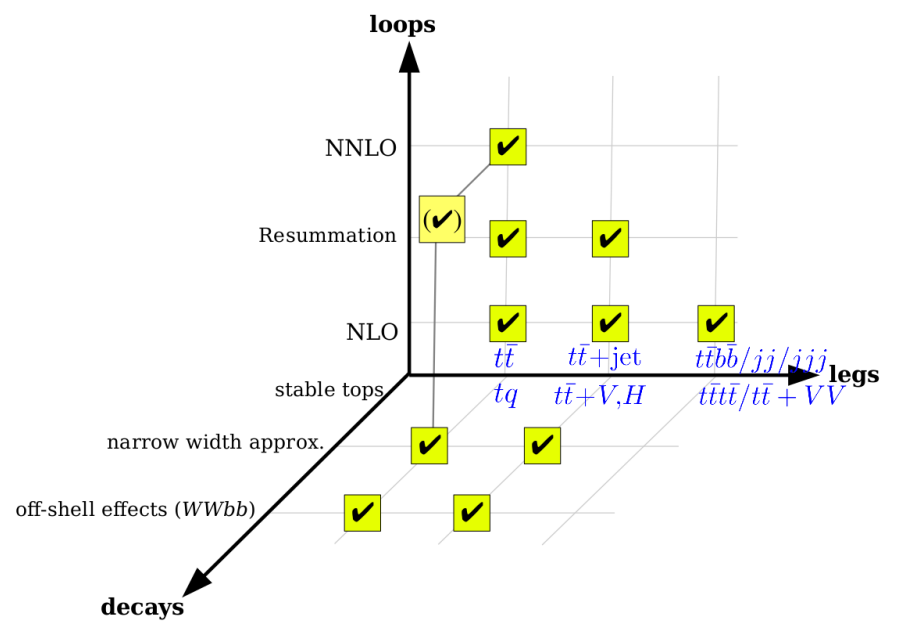

Figure 1: Three dimensions of progress in top quark theory and the current state-of-the-art.

Data taking at the Large Hadron Collider (LHC) is in full swing. If the collider experiment continues its current performance, it will conclude run-I \& II (at $13 \mathrm{TeV}$ center-of-mass energy) with a luminosity yield of more than $150 \mathrm{fb}^{-1}$ by the end of 2018. The wealth of this data, already now, translates into impressive physics results by the detector experiments: A large variety of Standard Model (SM) processes has been observed with high significance, yielding deep insights into the fundamental interaction dynamics. Moreover, major parts of generic (or minimal) New Physics models have been excluded, putting severe constraints on modifications of the SM at a TeV scale. In the absence of striking signals of physics beyond the SM, the search for small deviations and subtle effects becomes increasingly important. This motivates a research program of precise and broad investigations that leave no stone unturned. Top quark physics is at the heart of these studies as it couples strongest to the Higgs boson and it is expected to be most sensitive to New Physics. Its unique decay properties allow accessing spin information and electroweak properties, in contrast to the other quarks, which hadronize.

In this talk I will review recent theory progress in top quark production, give a brief overview of the current state-of-the-art, and discuss how this progress translates into a better understanding of SM physics and beyond.

\section{State-of-the-art}

Top quark theory made significant progress in recent years and I will try to organize these advancements in three directions, see Fig. 1. The first direction involves predictions at higher orders (loops) in perturbation theory, the second direction involves multi-particle final states (legs), and the third is about the realistic description of top quark final states (decays). As can be seen from Fig. 1, much progress has been made already and top quark theory is in very good shape: The standard candle processes $p p \rightarrow t \bar{t}$ and $p p \rightarrow t j$ are available at next-to-next-to leading order (NNLO) precision, pair production is known for stable top quarks, and single top quark production is known including top quark decays (moving into the third dimension in Fig. 1). At next-to leading order (NLO) these processes have even been calculated including the description of non-resonant effects. 

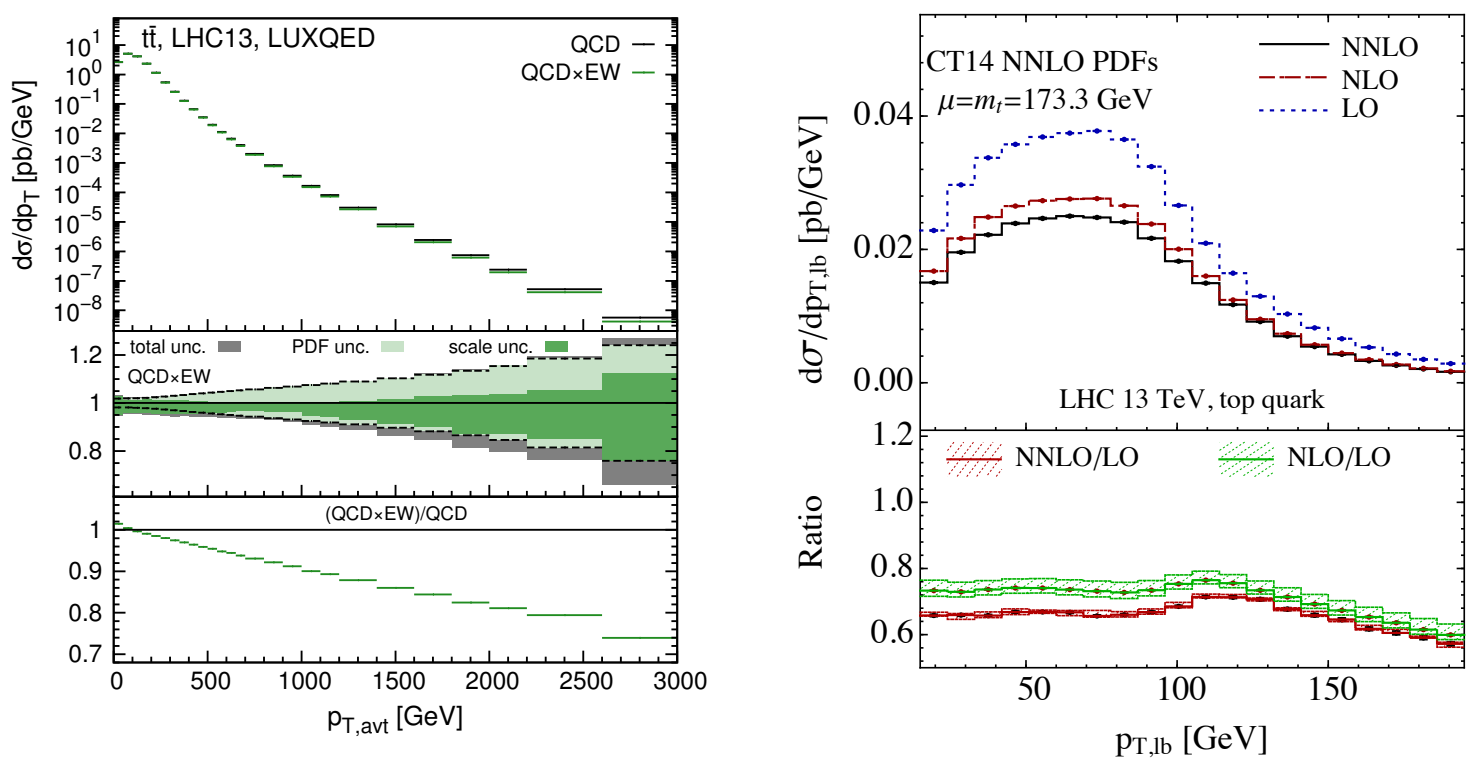

Figure 2: Left: NNLO QCD plus NLO EW corrections for the transverse momentum distribution of a top quark [1]. Right: NNLO QCD correction of single top quark production and decay for the lepton $b$-jet transverse momentum [11].

Processes with additional final state particles are known at least through NLO Quantum Chromodynamics (QCD), for some processes resummation and electroweak effects are known, for others the description of top quark decays have been included at NLO. In the following, I will discuss topics at the edges of this diagram which represent some most recent works (of the last 1-2 years) that contributed to advancing the state-of-the-art.

In Ref. [1] the authors present predictions for $p p \rightarrow t \bar{t}$ at the LHC which combines the NNLO QCD calculation with NLO electroweak (EW) corrections. While the separate calculations were achieved already several years ago [2-5], the consistent combination was done in Ref. [1] for the first time. The results allow for a precise estimate of the remaining perturbative uncertainties in the SM (see middle pane of Fig. 2, left). Precision QCD predictions are supplemented with the correct high energy behaviour due to the inclusion of electroweak Sudakov logarithms of the form $\alpha \log \left(s_{i j} / M_{W}^{2}\right)$. At top quark transverse momenta of $2 \mathrm{TeV}$ this Sudakov suppression amounts up to $-20 \%$ (see lower pane of Fig. 2, left). This weak virtual correction is partly compensated by $+3 \%$ from real weak emission corrections at high energies. In addition, the authors study uncertainties from parton distribution functions, different combinations of the $\alpha_{s}$ and $\alpha$ corrections, and the impact of photonic initial states.

A realistic prediction for top quark pair production needs to account for the decays of top quarks. The differential NNLO QCD corrections to production [2] and decay [6,7] are known for several years. Yet, their combination into one complete prediction is still outstanding. A convenient framework allowing the inclusion of highest perturbative orders is the narrow-width approximation (NWA). It systematically separates dynamics into a production and decay contribution through a 
parametric approximation in $\Gamma_{t} / m_{t} \approx 0.8 \%$. A first step towards a complete result for $t \bar{t}$ production is presented in Ref. [8], where the authors combine approximate NNLO results for the production process and the exact NNLO corrections for the decay in the NWA. This allows them to present fully differential results for a realistic final state with leptons, jet and missing transverse energy, including the detector cuts as considered by ATLAS and CMS. A second step towards the exact calculation has been made in Ref. [9], which includes the helicity dependent two-loop amplitudes for hadronic $t \bar{t}$ production. This is one major building block besides the double-unresolved real corrections, the real-virtual corrections and the mixed production-decay corrections.

The state-of-the-art is even more advanced when it comes to single top quark production. Here, a combination of production and decay at NNLO QCD in the NWA has been presented by the authors of Ref. [10]. Most recently, they presented fully differential results for the LHC [11] (see Fig. 2, right). While the cross section for single top quark production is about a third the size of $t \bar{t}$ production, millions of single top quark events will be produced and the physics analyses will significantly profit from the highly accurate predictions of Ref. [11].

When it comes to adding resummed contributions to fixed-order calculations, several new results appeared in the last few years. For $t \bar{t}$ production a double resummation at the next-tonext-to-leading logarithmic (NNLL) order has been combined with the NNLO QCD predictions in Ref. [12]. In this work, the authors combine NNLL resummation of threshold logarithms from soft-gluon emission with small mass logarithms in the boosted regime. Another recent work is Ref. [13], which includes the transverse momentum resummation for top quark pair production up to NLL at NLO QCD. The authors of Refs. [12] present the $M_{t \bar{t}}$ and $p_{\mathrm{T}, t}$ differential distributions, the authors of Refs. [13] present the $q_{\mathrm{T}}^{t \bar{t}}$ distribution. A new direction has been opened up by the works [14-18] as they tackle $2 \rightarrow 3$ processes, for the first time. Processes under consideration are top quark pair production in association with a Higgs boson [16, 17], $Z$ boson [15] and $W$ boson $[14,18]$. All calculations supplement NLO QCD predictions with the NLL soft-gluon resummation. See Fig. 3, left for $p p \rightarrow t \bar{t} H$ [16] as an example representing the reduction in unphysical scale dependence when the NLL resummation is added.

Another direction of progress in recent years concerns the NLO description of realistic $t \bar{t}$ final states beyond the NWA. These predictions require the calculation of (at least) $2 \rightarrow 6$ matrix elements for a final state of e.g. $b \bar{b} \ell^{+} v \ell^{-} \bar{v}$, where intermediate top quark resonances can be present but are not required. A description at NLO leads to the inclusion of non-factorizable effects (interaction between top quark decay products and the rest of the process), off-shell effects (top quarks far off their mass shell), and the irreducible background (diagrams with one or zero intermediate top quarks). Since the final state of all these contributions is the same, also their interference is included in the prediction.

Most recently, the results of Refs. [19,20] and Ref. [21] have appeared that show the remarkable capabilities of modern computational tools. In Ref. [20], the authors compute NLO off-shell effects to the final state $b \bar{b} \ell^{+} v j j$, i.e. top quark pair production in the semi-leptonic channel. This work extends previous predictions which only considered the di-leptonic channel and contains the additional challenge of virtual gluon exchange between one of the light quarks (from a $W$ boson decay) 

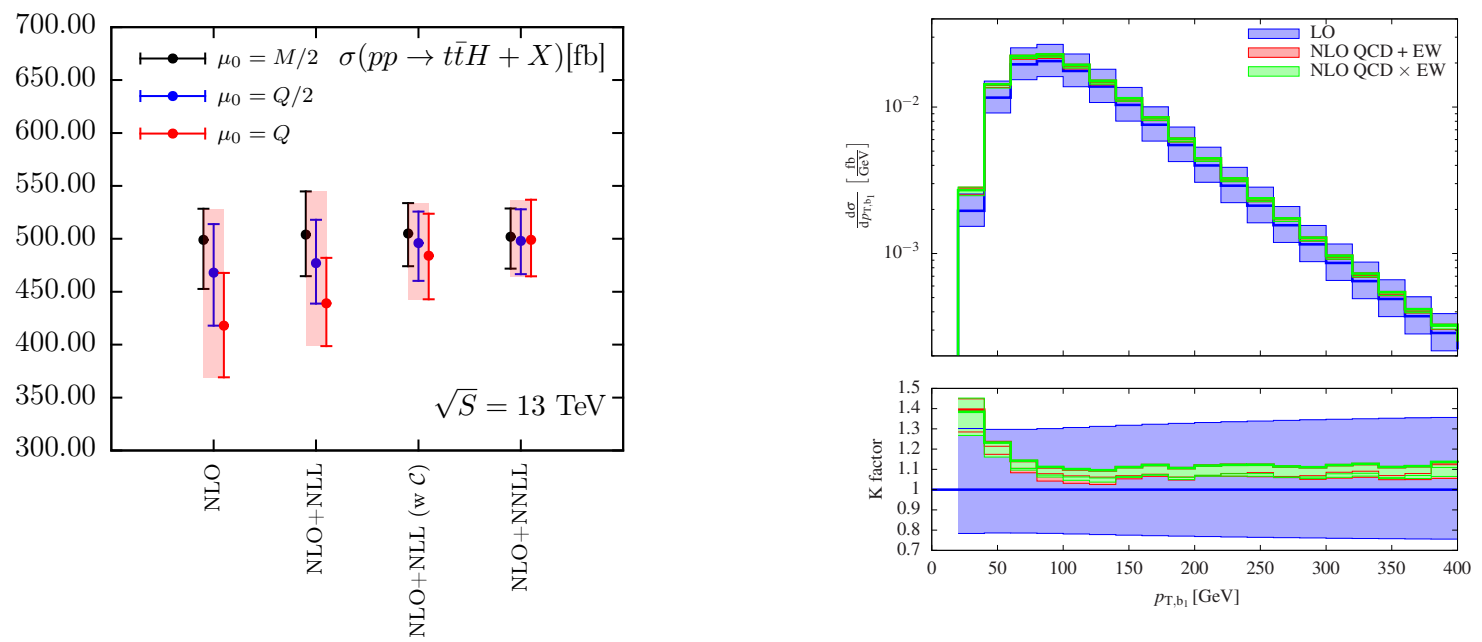

Figure 3: Left: Total cross sections and unphysical scale dependence for different resummation prescriptions of the $t \bar{t} H$ process [16]. Right: NLO $b$-jet transverse momentum distribution including off-shell effects and different QCD-EW combination schemes [19].

and the initial state. Ref. [21] deals with off-shell effects in associated top quark pair production with a photon. This process is of particular interest for measurements of the top quark electroweak couplings and searches for New Physics. The technical challenge is the additional photon in the final state which requires the calculation of one-loop seven-point functions and a large number of Feynman diagrams. Also in Ref. [19] the authors consider off-shell effects for associated top quark pair production, however with a Higgs boson. Here, the authors extend their earlier work at NLO QCD [22] by the inclusion of electroweak corrections, which even requires the calculation of one-loop eight-point functions. The effects of additive and multiplicative combination schemes are discussed, see Fig. 3, right. In all these works [19-21] the contribution of off-shell effects have been estimated to below a few percent for the total cross section which is consistent with the parametric approximation $\mathscr{O}\left(\Gamma_{t} / m_{t}\right)$ of the NWA. This has to be compared with typical NLO scale uncertainties of about $10 \%$. However, in certain edges of phase space, in particular in the vicinity of kinematic bounds, this estimate can be violated as demonstrated e.g. in Fig. 8 of Ref. [19].

\section{Physics impact on SM and BSM analysis}

Let me now discuss some examples of how novel precision calculations have advanced physics analyses at the LHC. The top quark mass determination is certainly one hot topic. The top quark mass $m_{t}$ is an important parameter of the SM and its precise value has implications for the stability of the electroweak vacuum. Current direct measurements [23] yield $m_{t}=173.0 \mathrm{GeV}$ with an uncertainty of $400 \mathrm{MeV}( \pm 0.2 \%)$. It is a long-standing discussion how well this extracted value corresponds to a properly defined mass parameter such as the on-shell mass, or some ill-defined "Monte Carlo mass" (see e.g. Ref. [24]). The main issue is that first principle quantum field theory predictions are often interfaced to parton showers for the subsequent detector simulation. These parton showers involve a certain degree of modelling and mix-up of perturbative orders which diminish the well-defined mass definition. It is surely impossible to completely remove all modelling 

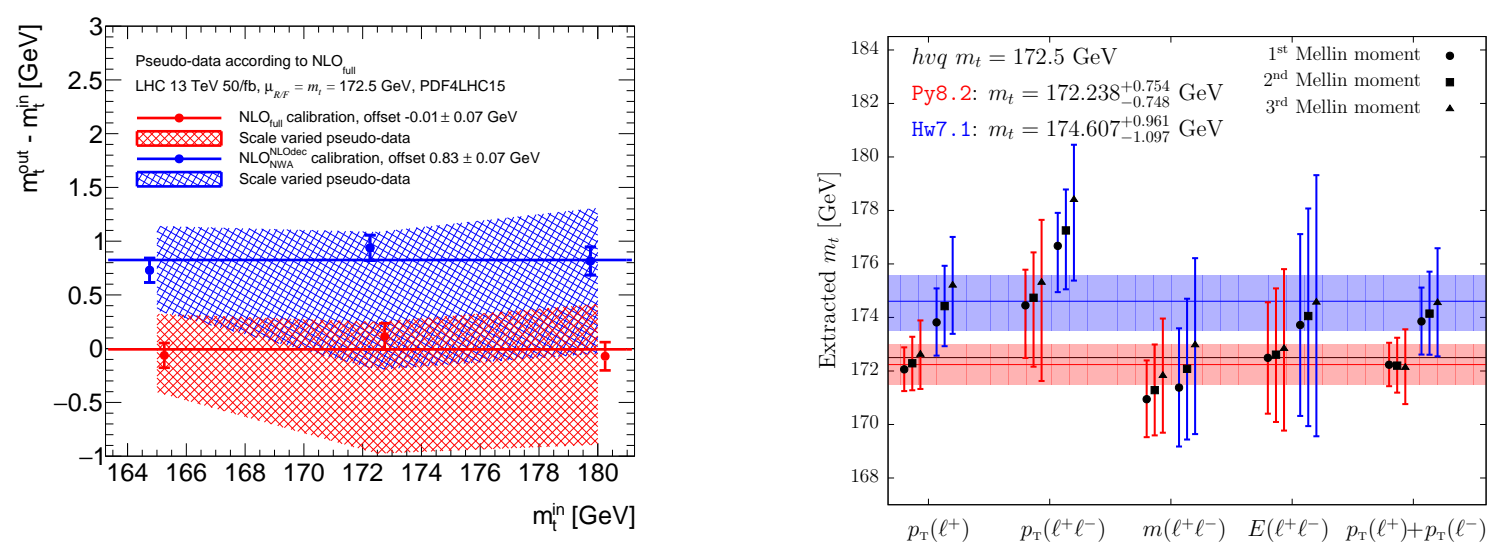

Figure 4: Left: Offset between input and extracted top quark mass arising from off-shell effects and the corresponding scale uncertainty bands [25]. Right: Comparison of extracted top quark masses from different parton shower models and leptonic observables, including uncertainty error bars [26].

at the interface between experiment and theory. However, one should try to reduce the amount of modelling by advancing first principle calculations. This requires pushing the perturbative orders, a realistic description of the process dynamics, and an understanding of non-perturbative contributions (renormalons and other). Some of these points have been addressed in the following recent publications.

In Ref. [25] the authors investigate the impact of different NLO QCD descriptions of $t \bar{t}$ production on the top quark mass extraction from differential measurements. In particular, $m_{t}$ extractions from the $m_{\ell b}$ and $m_{\mathrm{T} 2}$ observables are considered using a complete off-shell calculation, which is compared to a NWA calculation with all spin correlations and one that is interfaced to a parton shower. This comparison allows answering questions about the influence of off-shell and interference effects on $m_{t}$ extractions, as well as the offset when a parton shower is used to describe the top quark decays. As an example, the left plot of Fig. 4 shows the offset in $m_{t}$ that can be attributed to off-shell effects (solid lines), including the corresponding scale uncertainty (bands). The impact of finite-width effects in $t \bar{t}$ production has also been studied in Ref. [26], however, with a slightly different focus. The authors study sets of purely leptonic observables such as $p_{\mathrm{T}}^{\ell}, m_{\ell^{+} \ell^{-}}$or $E_{\ell^{+} \ell^{-}}$, as well as the peak of the $b$-jet energy distribution. In contrast to Ref. [25], this work presents parton shower interfaces to both, the NWA calculation and the full calculation with finite-width effects, and tests different parton showers. Fig. 4, right shows some representative findings for different observables and parton shower types. A sizable discrepancy between predictions from Pythia and Herwig can be observed and the authors argue that the difference has to be interpreted as theoretical uncertainty. A third recent work which presents top quark mass studies is Ref. [27]. The considered final state is $t \bar{t}+$ jet and the main observable of study is the normalised inverse invariant mass distribution. Similar to the works on $t \bar{t}$, a full $b \bar{b} \ell^{-} \bar{v} \ell^{+} v+$ jet calculation, including all finite-width effects, is compared to results of a NWA calculation to estimate off-shell effects on the $m_{t}$ determination. Interestingly, NLO corrections to the top quark decays turn out to be crucial for the correct description of the process. 

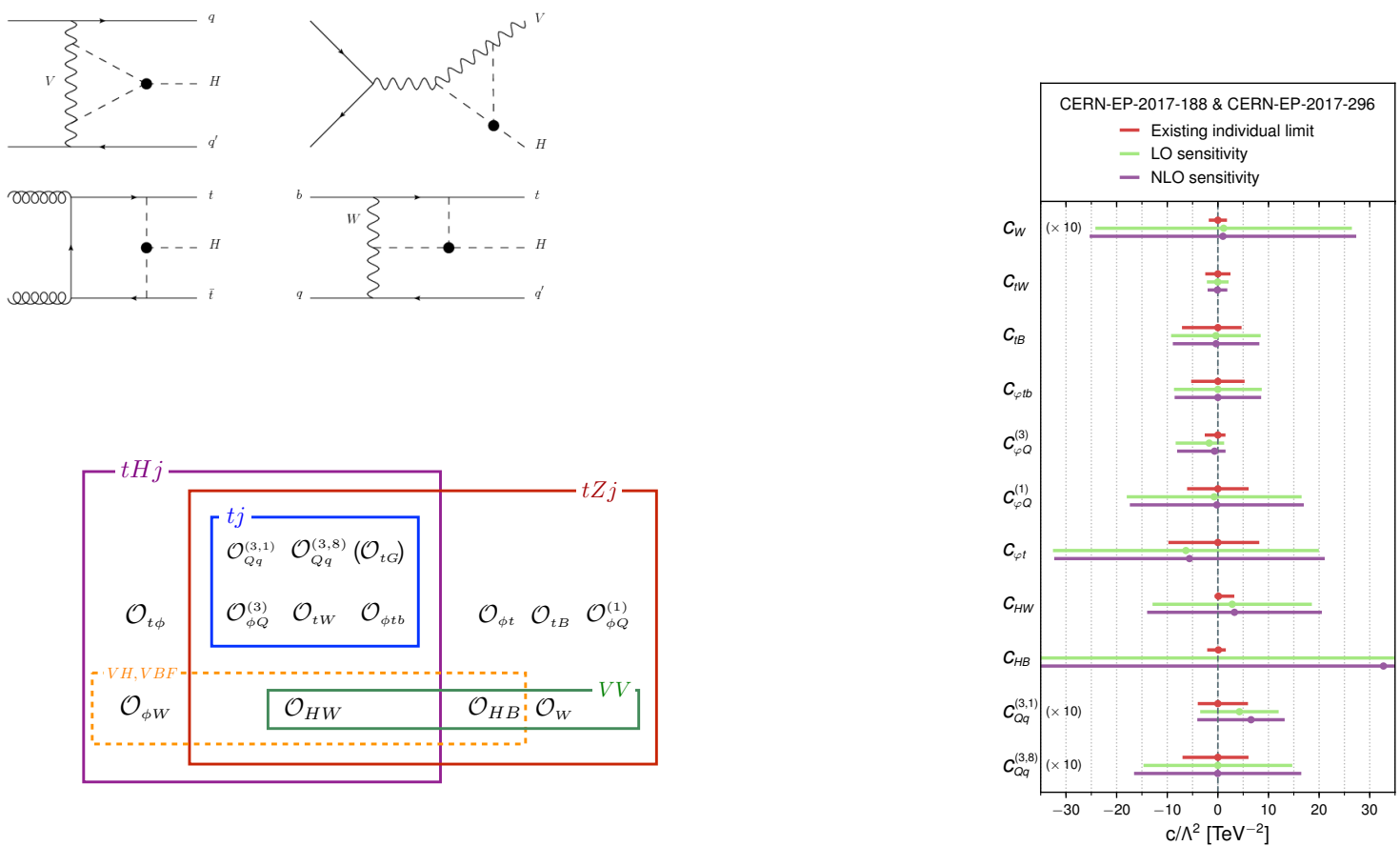

Figure 5: Top left: Loop diagrams yielding sensitivity to the Higgs boson self-coupling [29]. Bottom left: Dimension-six operators entering different top quark processes and weak boson production [28]. Right: Constraints on various dimension-six coefficients from measurements of single top quark $+Z$ boson production at the LHC.

Let us now put a focus on probing physics beyond the SM in the top quark sector. The authors of Ref. [28] study single top quark production in association with a Higgs or $Z$ boson, allowing for the influence of dimension-six operators from heavy new physics. One interesting feature in these processes is a numerical cancellation due to destructive interference between diagrams where the external boson couples either to a gauge boson or a fermion. This destructive interference in the SM process is largely eliminated when new physics effects enter the coupling. Hence, the cross section increases and yields strong sensitivity to new interactions. Another interesting feature is illustrated in Fig. 5 (bottom left): certain dimension-six operators are shared between different processes. For example, the operator $\mathscr{O}_{H W}$ enters the processes $p p \rightarrow t H j, p p \rightarrow t Z j$ as well as vector boson fusion/scattering and $p p \rightarrow Z H$. Ref. [28] describes how the interplay between these processes can be utilized. Some possible constraints are shown on the right of Fig. 5.

Probing the Higgs boson self-coupling $\lambda$ through the cubic interaction is a well-known challenge at the LHC. Direct studies in the di-Higgs production process $p p \rightarrow H H$ suffer from its very small cross section. An alternative way to probe the self-coupling has been presented in Ref. [29]. Their proposal is to use associated production processes such as $t \bar{t}+H$ or $Z+H$ to obtain sensitivity to $\lambda$ through electroweak virtual corrections (see Fig. 5, top left). The results show that the future high-luminosity LHC run will allow to put constraints on the coupling. Whether these constraints 

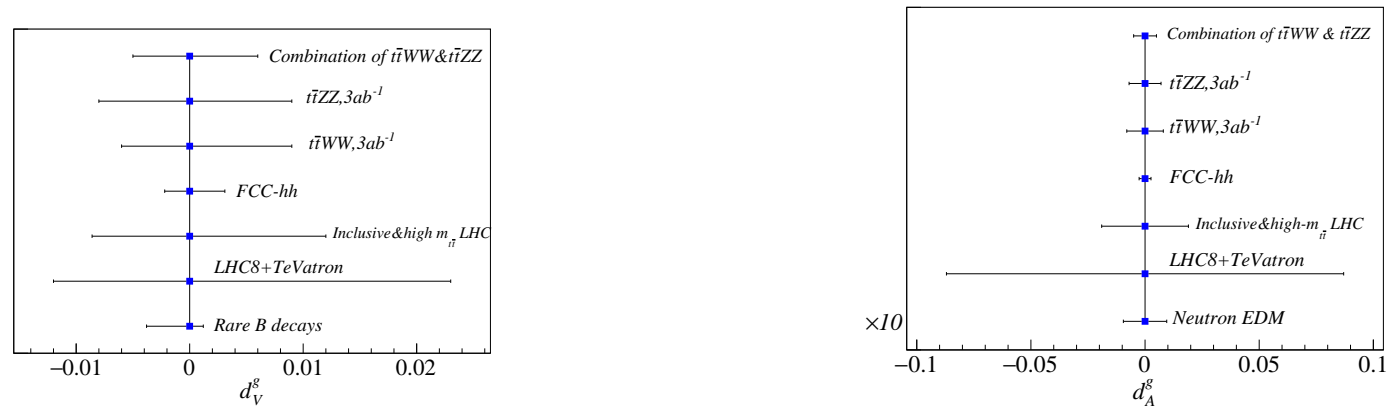

Figure 6: Constraints on weak magnetic (left) and electric (right) dipole couplings from Ref. [30].

will be competitive with the ones from direct di-Higgs boson production strongly depends on the expected experimental and theoretical uncertainties and is still an open question.

Finally, we look at probing the top quark electroweak couplings, i.e. the interaction of top quarks with weak gauge bosons. The recent investigation of Ref. [30] studies top quark pairs produced in association with pairs of $Z$ and $W$ bosons. The authors put special emphasis on constraining the weak dipole moments of the top quark and consider total cross sections as well as differential distributions. For the cleanest decay channels involving only leptons, they find constraints that are comparable with the prospective ones from $t \bar{t}+Z$ production at the LHC. In Fig. 6, also a comparison to constraints from rare $B$-decays and neutron electric-dipole moments is shown.

\section{References}

[1] M. Czakon, D. Heymes, A. Mitov, D. Pagani, I. Tsinikos and M. Zaro, JHEP 1710 (2017) 186 doi:10.1007/JHEP10(2017)186 [arXiv:1705.04105 [hep-ph]].

[2] M. Czakon, P. Fiedler and A. Mitov, Phys. Rev. Lett. 110 (2013) 252004 doi:10.1103/PhysRevLett.110.252004 [arXiv:1303.6254 [hep-ph]].

[3] W. Beenakker, A. Denner, W. Hollik, R. Mertig, T. Sack and D. Wackeroth, Nucl. Phys. B 411 (1994) 343. doi:10.1016/0550-3213(94)90454-5

[4] J. H. Kühn, A. Scharf and P. Uwer, Eur. Phys. J. C 51 (2007) 37 doi:10.1140/epjc/s10052-007-0275-x [hep-ph/0610335].

[5] W. Bernreuther, M. Fücker and Z. G. Si, Phys. Lett. B 633 (2006) 54 Erratum: [Phys. Lett. B 644 (2007) 386] doi:10.1016/j.physletb.2006.11.052, 10.1016/j.physletb.2005.11.056 [hep-ph/0508091].

[6] J. Gao, C. S. Li and H. X. Zhu, Phys. Rev. Lett. 110 (2013), 042001 doi:10.1103/PhysRevLett.110.042001 [arXiv:1210.2808 [hep-ph]].

[7] M. Brucherseifer, F. Caola and K. Melnikov, JHEP 1304 (2013) 059 doi:10.1007/JHEP04(2013)059 [arXiv:1301.7133 [hep-ph]].

[8] J. Gao and A. S. Papanastasiou, Phys. Rev. D 96 (2017), 051501 doi:10.1103/PhysRevD.96.051501 [arXiv:1705.08903 [hep-ph]].

[9] L. Chen, M. Czakon and R. Poncelet, JHEP 1803 (2018) 085 doi:10.1007/JHEP03(2018)085 [arXiv:1712.08075 [hep-ph]]. 
[10] E. L. Berger, J. Gao, C.-P. Yuan and H. X. Zhu, Phys. Rev. D 94 (2016), 071501 doi:10.1103/PhysRevD.94.071501 [arXiv:1606.08463 [hep-ph]].

[11] E. L. Berger, J. Gao and H. X. Zhu, JHEP 1711 (2017) 158 doi:10.1007/JHEP11(2017)158 [arXiv:1708.09405 [hep-ph]].

[12] M. Czakon, A. Ferroglia, D. Heymes, A. Mitov, B. D. Pecjak, D. J. Scott, X. Wang and L. L. Yang, JHEP 1805 (2018) 149 doi:10.1007/JHEP05(2018)149 [arXiv:1803.07623 [hep-ph]].

[13] S. Catani, M. Grazzini and H. Sargsyan, arXiv:1806.01601 [hep-ph].

[14] A. Broggio, A. Ferroglia, G. Ossola and B. D. Pecjak, JHEP 1609 (2016) 089 doi:10.1007/JHEP09(2016)089 [arXiv:1607.05303 [hep-ph]].

[15] A. Broggio, A. Ferroglia, G. Ossola, B. D. Pecjak and R. D. Sameshima, JHEP 1704 (2017) 105 doi:10.1007/JHEP04(2017)105 [arXiv:1702.00800 [hep-ph]].

[16] A. Kulesza, L. Motyka, T. Stebel and V. Theeuwes, Phys. Rev. D 97 (2018), 114007 doi:10.1103/PhysRevD.97.114007 [arXiv:1704.03363 [hep-ph]].

[17] A. Broggio, A. Ferroglia, M. C. N. Fiolhais and A. Onofre, Phys. Rev. D 96 (2017), 073005 doi:10.1103/PhysRevD.96.073005 [arXiv:1707.01803 [hep-ph]].

[18] A. Kulesza, L. Motyka, D. Schwartländer, T. Stebel and V. Theeuwes, PoS EPS -HEP2017 (2017) 465 doi:10.22323/1.314.0465 [arXiv:1710.06810 [hep-ph]].

[19] A. Denner, J. N. Lang, M. Pellen and S. Uccirati, JHEP 1702 (2017) 053 doi:10.1007/JHEP02(2017)053 [arXiv:1612.07138 [hep-ph]].

[20] A. Denner and M. Pellen, JHEP 1802 (2018) 013 doi:10.1007/JHEP02(2018)013 [arXiv:1711.10359 [hep-ph]].

[21] G. Bevilacqua, H. B. Hartanto, M. Kraus, T. Weber and M. Worek, arXiv:1803.09916 [hep-ph].

[22] A. Denner and R. Feger, JHEP 1511 (2015) 209 doi:10.1007/JHEP11(2015)209 [arXiv:1506.07448 [hep-ph]].

[23] M. Tanabashi et al. [ParticleDataGroup], Phys. Rev. D 98 (2018), 030001. doi:10.1103/PhysRevD.98.030001

[24] P. Nason, arXiv:1712.02796 [hep-ph].

[25] G. Heinrich, A. Maier, R. Nisius, J. Schlenk, M. Schulze, L. Scyboz and J. Winter, JHEP 1807 (2018) 129 doi:10.1007/JHEP07(2018)129 [arXiv:1709.08615 [hep-ph]].

[26] S. Ferrario Ravasio, T. Ježo, P. Nason and C. Oleari, Eur. Phys. J. C 78 (2018), 458 doi:10.1140/epjc/s10052-018-5909-7 [arXiv:1801.03944 [hep-ph]].

[27] G. Bevilacqua, H. B. Hartanto, M. Kraus, M. Schulze and M. Worek, JHEP 1803 (2018) 169 doi:10.1007/JHEP03(2018)169 [arXiv:1710.07515 [hep-ph]].

[28] C. Degrande, F. Maltoni, K. Mimasu, E. Vryonidou and C. Zhang, [arXiv:1804.07773 [hep-ph]].

[29] F. Maltoni, D. Pagani, A. Shivaji and X. Zhao, Eur. Phys. J. C 77 (2017), 887 doi:10.1140/epjc/s10052-017-5410-8 [arXiv:1709.08649 [hep-ph]].

[30] S. M. Etesami, S. Khatibi and M. Mohammadi Najafabadi, Phys. Rev. D 97 (2018), 075023 doi:10.1103/PhysRevD.97.075023 [arXiv:1712.07184 [hep-ph]]. 